\title{
Effects of a Novel Synthetic Vitamin A-Like Compound (a Polyprenoic Acid Derivative, E-5166) on Neutrophil- Derived Reactive Oxygen Species
}

\author{
Yoshiki MiYachi, ${ }^{1, *}$ Sadao Imamura,${ }^{1}$ and Yukie NiWA ${ }^{2}$ \\ ${ }^{1}$ Department of Dermatology, Faculty of Medicine, Kyoto University, \\ Kyoto 606, Japan \\ ${ }^{2}$ Niwa Institute for Immunology, Tosashimizu 787-03, Japan
}

(Received February 1, 1988)

\begin{abstract}
Summary In order to elucidate the mechanisms of anti-inflammatory activity of a new synthetic vitamin A-like compound (polyprenoic acid derivative, E-5166), the anti-oxidant effect of the agent was evaluated by examining the levels of reactive oxygen species both in the zymosanstimulated neutrophil system and in the cell-free, xanthine-xanthine oxidase system. E-5166 inhibited the generation of hydroxyl radicals but had little effect on superoxide, hydrogen peroxide, or chemiluminescence when used at therapeutic concentrations. The favorable effects of E-5166 on several inflammatory skin disorders are discussed in connection with the anti-oxidant activity of the drug.
\end{abstract}

Key Words: E-5166, reactive oxygen species, neutrophils, anti-inflammatory effect

Vitamin A and its synthetic analogs (retinoids) have been demonstrated to affect cellular differentiation and proliferation with resultant anti-neoplastic effects. In the field of dermatology, retinoids have been used for the treatment of not only keratinizing disorders but also several cutaneous inflammations such as pustular psoriasis and cystic acne, where neutrophils play an important role. These effects have been attributed to the anti-inflammatory action by retionids $[1,2]$, however, the exact mechanism by which retinoids work in the inflammatory process still remains unclear.

Recently we reported that the anti-inflammatory effects of retinoids can be partly explained by their inhibition of reactive oxygen species (ROS) generation by neutrophils [3]. In the present investigation, effects of a new synthetic vitamin

*To whom correspondence should be addressed. 
A-like compound, E-5166, on ROS levels were examined and compared with those of tretinoin (all-trans-retinoic acid).

\section{MATERIALS AND METHODS}

Chemicals. Tretinoin (all-trans-retinoic acid) was purchased from Sigma Chemical Co. (St. Louis, MO). E-5166 was a generous gift from Eisai Co., Ltd., Tokyo. All other chemicals were analytical reagent grade.

Preparation of neutrophils for ROS assays. Human neutrophils were prepared from heparinized peripheral venous blood according to a previously described method [4]. After centrifugation of the blood over a Ficoll-Hypaque gradient, the cell pellet containing neutrophils and erythrocytes was washed with saline solution and resuspended in plasma containing dextran 170 (molecular mass, 170,000 daltons) at a final concentration of $1 \%$. The neutrophils were recovered after sedimentation at unit gravity, and the few contaminating erythrocytes were lysed by treating the preparation with $0.876 \% \mathrm{NH}_{4} \mathrm{Cl}$. The neutrophils were then resuspended in Krebs-Ringer phosphate buffer (KRP) [5] containing $5 \mathrm{~mm}$ glucose and $1 \mathrm{mg} / \mathrm{ml}$ gelatin for the ROS assays. Gelatin was added in order to prevent the neutrophils from adhering to the test tubes. Gelatin was excluded from the medium used in the $\mathrm{OH}^{\cdot}$ generation assay on account of its inhibitory effect.

$R O S$ generation assays. Opsonized zymosan (Sigma) was freshly prepared before each experiment by incubating $11 \mathrm{mg}$ of zymosan with $1 \mathrm{ml}$ of autologous serum for $30 \mathrm{~min}$ at $37^{\circ} \mathrm{C}$. The formation of $\mathrm{O}_{2}{ }^{-}$was determined according to the method of Johnston and Lehmeyer [6]. Neutrophils $\left(4 \times 10^{6}\right)$ were preincubated at $37^{\circ} \mathrm{C}$ for $10 \mathrm{~min}$ with opsonized zymosan at $1 \mathrm{mg} / \mathrm{ml}$. After the addition of $0.1 \mathrm{~mm}$ ferricytochrome $c$ (type III; Sigma), the neutrophils were incubated for a further $30 \mathrm{~min}$ at $37^{\circ} \mathrm{C}$. Immediately after the neutrophils and opsonized zymosan had been sedimented by centrifugation, the supernatants were assayed for superoxide dismutase (SOD) inhibitable ferricytochrome $c$ reduction by measuring their absorbance at $550 \mathrm{~nm}$ with a spectrophotometer (Hitachi, Tokyo). In order to confirm the specificity of the $\mathrm{O}_{2}{ }^{-}$assay, SOD was added at a concentration of 100 units $/ \mathrm{ml}$, and the inhibition of cytochrome $c$ reduction by $\mathrm{O}_{2}{ }^{-}$was measured.

The generation of $\mathrm{H}_{2} \mathrm{O}_{2}$ was measured by quantitating the decrease in the fluorescence intensity of scopoletin (Sigma) resulting from its peroxidase-mediated oxidation by $\mathrm{H}_{2} \mathrm{O}_{2}$ [7]. After the incubation of $2.5 \times 10^{6}$ neutrophils in KRP with $1 \mathrm{mg} / \mathrm{ml}$ of opsonized zymosan for $10 \mathrm{~min}$ at room temperature, $0.1 \mathrm{ml}$ of $50 \mathrm{~mm}$ scopoletin in KRP and $0.1 \mathrm{ml}$ of $1 \mathrm{mg} / \mathrm{ml}$ horseradish peroxidase (type II; Sigma) in phosphate-buffered saline (PBS) were added. The rate of decrease of the fluorescence intensity of the scopoletin over a 30 -min period was quantitated using a fluorescence spectrophotometer (Hitachi). Excitation and emission wavelength are 360 and $460 \mathrm{~nm}$, respectively.

The generation of $\mathrm{OH}^{-}$was quantitated from the amount of ethylene gas formed by the reaction of $\alpha$-ketomethiol butylic acid (KMB; Sigma) with $\mathrm{OH}$. 
generated by neutrophils [8]. Neutrophils $\left(2 \times 10^{6}\right)$ in $2 \mathrm{ml} \mathrm{KRP} \mathrm{were} \mathrm{preincubated}$ with $1 \mathrm{~mm} \mathrm{KMB}$ for $5 \mathrm{~min}$ at $37^{\circ} \mathrm{C}$. After $(1 \mathrm{mg} / \mathrm{ml})$ opsonized zymosan had been added, the incubation was continued for a further $10 \mathrm{~min}$. The amount of ethylene gas formed was assayed at 10, 20, and 30 min using a gas chromatograph (Hitachi), and the total of these measurements served as the $\mathrm{OH}^{\cdot}$ value.

Chemiluminescence was measured in a scintillation spectrometer (Packard IL) according to the method of Allen and Loose [9] with slight modifications. Neutrophils $\left(5 \times 10^{6}\right)$ in $3 \mathrm{ml}$ of colorless Hanks' solution containing gelatin were incubated for $10 \mathrm{~min}$ at $37^{\circ} \mathrm{C}$ with opsonized zymosan in the absence of luminol. The chemiluminescence was monitored using a spectrometer operated in the out-ofcoincidence summation mode. All procedures were performed in the dark.

Effects of E-5166 and tretinoin on the generation of ROS by neutrophils. The agents were first dissolved in dimethylsulfoxide (DMSO), and this solution was then added to the neutrophil-containing medium of each ROS assay system to make final concentrations of 1,10 , and $100 \mu \mathrm{M}$. The final concentration of DMSO was $0.1 \%$. The same volume of DMSO was added to the control medium. Since DMSO is a potent radical scavenger and the generation of ROS in the controls was reduced by the addition of DMSO, the effect of the agent on ROS generation was calculated by using the control values after the addition of DMSO.

Effects of E-5166 and tretinoin on ROS generation in the xanthine-xanthine oxidase system. In separate experiments, the effects of the agents on ROS generation were investigated in the xanthine-xanthine oxidase system. Instead of adding neutrophils and opsonized zymosan, $0.1 \mathrm{ml}$ of hypoxanthine solution $(13.5 \mathrm{mg}$ in $50 \mathrm{ml}$ physiological saline) and $0.05 \mathrm{ml}$ of $50 \mathrm{~mm}$ ethylenediaminetetraacetate (EDTA) were diluted in $2 \mathrm{ml} \mathrm{KRP} \mathrm{(pH} \mathrm{7.2-7.4).} \mathrm{To} \mathrm{generate} \mathrm{O}_{2}{ }^{-}, 0.1 \mathrm{ml}$ of 0.12 unit/ml dialyzed xanthine oxidase was added, and the amount of each ROS was determined as already described.

\section{RESULTS}

As shown in Fig. 1, tretinoin reduced the $\mathrm{O}_{2}{ }^{-}$formation dose-dependently in both cellular and cell-free ROS generation systems. However, no significant effect was observed with E-5166 in either neutrophil or xanthine-xanthine oxidase system.

The level of $\mathrm{H}_{2} \mathrm{O}_{2}$ was also decreased by tretinoin in both assay systems in a dose-related way (Fig. 2). Only the highest concentration of E-5166 inhibited the $\mathrm{H}_{2} \mathrm{O}_{2}$ generation by neutrophils, and no significant reduction in $\mathrm{H}_{2} \mathrm{O}_{2}$ level was noticed in a cell-free system by E-5166 at any concentration tested.

As demonstrated in Fig. 3, $\mathrm{OH} \cdot$ levels were again reduced significantly in a dose-dependent manner by tretinoin in both ROS producing systems. On the contrary, E-5166 significantly inhibited the neutrophil-derived $\mathrm{OH} \cdot$ generation dose-dependently without affecting $\mathrm{OH}^{\cdot}$ levels in the xanthine-xanthine oxidase system. Chemiluminescence was affected by neither E-5166 nor tretinoin (Fig. 4). 


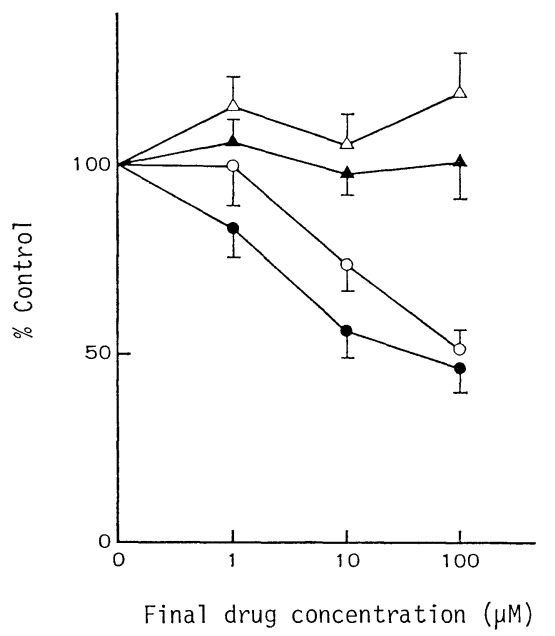

Fig. 1. Effects of E-5166 and tretinoin on $\mathrm{O}_{2-}$ levels. Each point denotes the mean of triplicate assays. Bars indicate SD. Means $\pm S D$ are given. Tretinoin: $\bigcirc$, neutrophil system; -, cell-free system. E-5166: $\triangle$, neutrophil system; $\mathbf{\Delta}$, cell-free system.

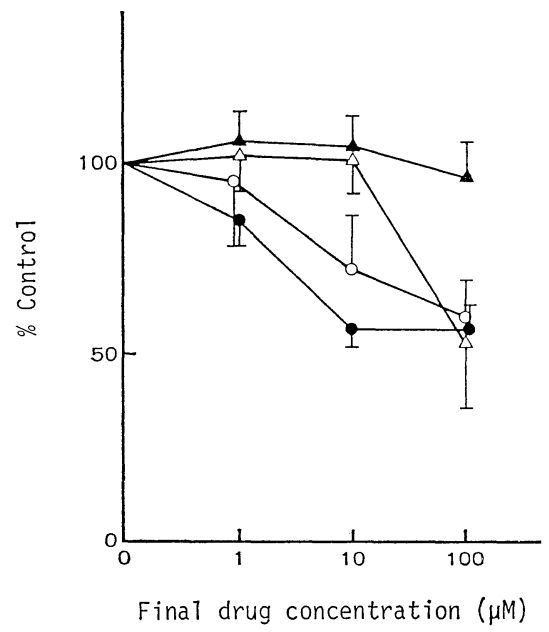

Fig. 2. Effects of E-5166 and tretinoin on $\mathrm{H}_{2} \mathrm{O}_{2}$ levels. For symbols, see the legend to Fig. 1.

\section{DISCUSSION}

E-5166 is a new synthetic vitamin A-like compound originally described by Muto and Omori [10] and Muto et al. [11] and has a strong in vitro binding affinity to intracellular binding proteins for acidic retinoids. It is clinically effective for various inflammatory skin diseases as well as hyperproliferative and dyskeratotic skin diseases. However, the exact mechanism of anti-inflammatory activity of 


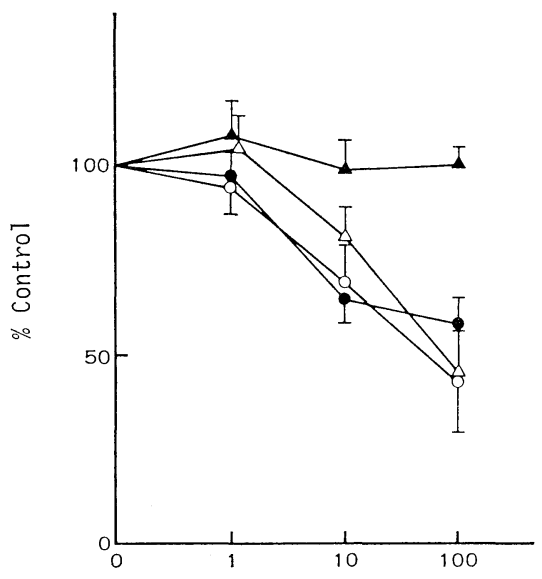

Final drug concentration $(\mu \mathrm{M})$

Fig. 3. Effects of E-5166 and tretinoin on $\mathrm{OH}^{\cdot}$ levels. For symbols, see the legend to Fig. 1.

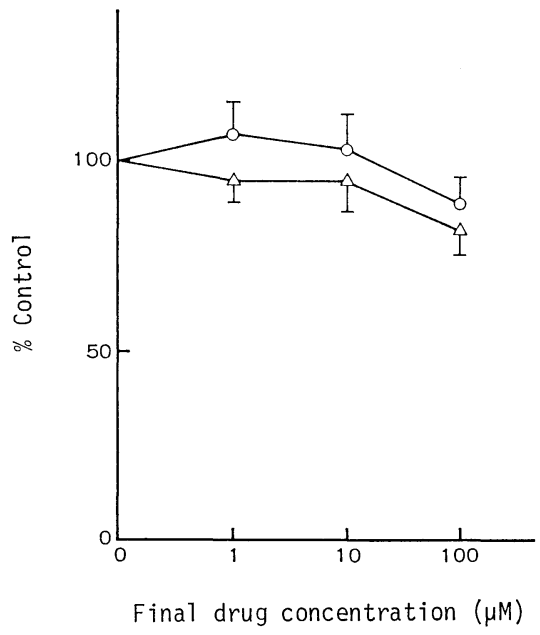

Fig. 4. Effects of E-5166 and tretinoin on chemiluminescence. For symbols, see the legend to Fig. 1.

E-5166 still remains to be studied. E-5166 is reported to inhibit the epidermal growth factor-stimulated arachidonic acid release through inhibition of the epidermal growth factor-stimulated phosphatidylinositol turn over, and thus this compound may have profound effects on phospholipid metabolism [12]. Previously we have reported anti-oxidant effects of retinoids and discussed a possible mechanism by which retinoids may scavenge ROS to exert their clinical effects on several cutaneous inflammatory disorders [3]. Since neutrophil-derived oxygen metabolites released at sites of inflammation induce tissue damage there [13], it is reasonable to speculate 
that the drugs with anti-oxidant activity may work as anti-inflammatory agents. The present study demonstrates that E-5166 inhibits $\mathrm{OH} \cdot$ generation in neutrophil system at the therapeutic levels and this inhibitory effect of E-5166 may partly account for its clinical effectiveness against inflammatory skin diseases.

\section{REFERENCES}

1. Plewig, G., and Wagner, A. (1981): Anti-inflammatory effects of 13-cis-retinoic acid. Arch. Dermatol. Res., 270, 89-94.

2. Camisa, C., Eisenstat, B., Ragaz, A., and Weissmann, G. (1982): The effects of retinoids on neutrophil functions in vitro. J. Am. Acad. Dermatol., 6, 620-629.

3. Yoshioka, A., Miyachi, Y., Imamura, S., and Niwa, Y. (1986): Anti-oxidant effects of retinoids on inflammatory skin diseases. Arch. Dermatol. Res., 278, 177-183.

4. Skosey, J.L., Chow, D.C., Damgaard, E., and Sorensen, L.B. (1973): Effects of cytochalasin B on the response of human polymorphonuclear leukocytes to zymosan. J. Cell Biol., 57, 237-240.

5. Cohen, P.P. (1957): Suspending media for normal tissues, in Monocytometric Technique and Tissue Metabolism, ed. by Umbreit, W.W., Burris, R.H., and Stauffer, J.F., Burgess Publishing, Minneapolis, pp. 149-150.

6. Johnston, R.B., Jr., and Lehmeyer, J.E. (1976): Elaboration of toxic oxygen by-products by neutrophils in a model of immune complex disease. J. Clin. Invest., 57, 836-841.

7. Root, R.K., Metcalf, J., Oshino, N., and Chance, B. (1975): $\mathrm{H}_{2} \mathrm{O}_{2}$ release from human granulocytes during phagocytosis. J. Clin. Invest., 55, 945-955.

8. Klebanoff, S.J., and Rosen, H. (1978): Ethylene formation by polymorphonuclear leukocytes. J. Exp. Med., 148, 490-506.

9. Allen, R.C., and Loose, L.D. (1976): Phagocytic activation of a luminol-dependent chemiluminescence in rat alveolar and peritoneal macrophages. Biochem. Biophys. Res. Commun., 69, 245-252.

10. Muto, Y., and Omori, M. (1981): A novel cellular retinoid-binding protein, F-type, in hepatocellular carcinoma. Ann. NY. Acad. Sci., 359, 91-103.

11. Muto, Y., Moriwaki, H., and Omori, M. (1981): In vitro binding affinity of novel synthetic polyprenoids (polyprenoic acids) to cellular retinol-binding proteins. Gann, 72, 974-977.

12. Suya, H., Aoyagi, T., Koizumi, H., Fukaya, T., and Nemoto, O. (1987): A novel synthetic vitamin A-like compound (a polyprenoic acid derivative, E-5166) inhibits the release of arachidonic acid stimulated by epidermal growth factor. J. Invest. Dermatol., 88, 630-633.

13. Miyachi, Y., Yoshioka, A., Imamura, S., and Niwa, Y. (1987): Polymorphonuclear leukocytederived reactive oxygen species in inflammatory skin diseases, in The Biological Role of Reactive Oxygen Species in Skin, ed. by Hayaishi, O., Imamura, S., and Miyachi, Y., the University of Tokyo Press, Tokyo, pp. 135-140. 